Article

\title{
Degree-Distance Based Topological Indices of Crystal Cubic Carbon Structure
}

\author{
Hong Yang ${ }^{1}$, Muhammad Kamran Siddiqui ${ }^{2, *}$, , Misbah Arshad ${ }^{2}$ and Muhammad Naeem ${ }^{3}$ \\ 1 School of Information Science and Engineering, Chengdu University, Chengdu 610106, China; \\ yanghong01@cdu.edu.cn \\ 2 Department of Mathematics, COMSATS University Islamabad, Sahiwal Campus 57000, Pakistan; \\ misbah_arshad15@yahoo.com \\ 3 Department of Mathematics, The University of Lahore, Pakpattan Campus 57400, Pakistan; \\ naeempkn@gmail.com \\ * Correspondence: kamransiddiqui75@gmail.com
}

Received: 27 October 2018; Accepted: 13 November 2018; Published: 15 November 2018

\begin{abstract}
Chemical graph theory comprehends the basic properties of an atomic graph. The sub-atomic diagrams are the graphs that are comprised of particles called vertices and the covalent bond between them are called edges. The eccentricity $\epsilon_{u}$ of vertex $u$ in an associated graph $G$, is the separation among $u$ and a vertex farthermost from $u$. In this article, we consider the precious stone structure of cubic carbon and registered Eccentric-connectivity index $\xi(G)$, Eccentric connectivity polynomial $\operatorname{ECP}(G, x)$ and Connective Eccentric index $C^{\tau}(G)$ of gem structure of cubic carbon for $n$-levels.
\end{abstract}

Keywords: degree; eccentricity; Eccentric-connectivity index $\xi(G)$; Eccentric connectivity polynomial $\operatorname{ECP}(G, x)$; Connective Eccentric index $C^{\xi}(G)$; crystal cubic carbon

\section{Introduction}

A branch of scientific chemistry is Chemical graph hypothesis, which relates to the nontrivial utilizations of graph hypothesis for taking care of the atomic issues. Its pioneers are Alexandru Balaban [1], Ante Graovac [2], Ivan Gutman [3], Milan Randić [4] and Nenad Trinajstić [5]. Chemical graph theory utilizes mathematical invariants to limit the structure of a particle into a solitary number which signifies the energy of molecule, structural sections, sub-atomic stretching and electronic structures. These graph theoretic invariants are utilized to connect with physical perceptions ascertained by experiments. In [6], chemical graph hypothesis ought to be seen not just as equivalent to different branches of theoretical chemistry but also as corresponding and essential for better comprehension of the idea of the compound structure.

There are sure synthetic intensifies that are valuable for the survival of living things. Carbon, oxygen, hydrogen and nitrogen are the primary components that aide in the creation of cells in the living things. Carbon is a fundamental component for human life. It is valuable in the development of proteins, starches and nucleic acids. It is imperative for the development of plants as carbon dioxide. The carbon particles can bond together in different ways, called allotropes of carbon. The notable structures are graphite and precious stone. As of late, numerous new structures have been found including nanotubes, buckminsterfullerene and sheets, precious stone cubic structure, and so on. The utilizations of various allotropes of carbon are talked about in detail in [7-9].

A molecular graph is a straight forward graph speaking to the carbon atom skeleton of a organic molecule. In the molecular graph, the vertices speak to the carbon molecules and the edges speak to the carbon-carbon bonds. 
Consider $G(V, E)$ as a fundamental basic graph, where $V$ and $E$ address the course of action of vertices and the game plan of edges. The amount of parts in $V$ is known as the request of the diagram $G$ and the amount of segments in $E$ is known as the extent of the chart $G$. The amount of vertices neighboring the vertex $v \in V$ is known as the level of $v$ and is connoted as $d_{v}$. On the off chance that no vertices in $u-v$ walk are reiterated, then it is called $u-v$ way in chart $G$. The length of a way is the amount of edges in it. The separation $d(u, v)$ from vertex $u$ to vertex $v$ is the length of a most constrained $u-v$ way in a chart $G$ where $u, v \in G$. In an associated diagram $G$, the flightiness $\epsilon_{u}$ of vertex $u$ is the separation among $u$ and a vertex most removed from $u$ in $G$.

A graph can be seen by a numeric number, a polynomial, a gathering of numbers or a system. A topological index (molecular structure descriptor) is a numerical regard related with manufactured constitution for relationship of substance structure with various physical properties, compound reactivity or natural activity. There are some noteworthy classes of topological files, for instance, separate-based topological lists, flightiness-based topological records, degree-based topological lists and tallying related polynomials and lists of diagrams.

The primary topological record is the Wiener list which was proposed in 1947 when Harold wiener [10] was taking a shot at the breaking point of paraffin. It was the main topological record which relied on the idea of separation between two vertices in a chart. Wiener considered it the way number. In any case, after that it was renamed as Wiener file and it is signified by $W$. The hypothesis of topological files began from the Wiener record. Wiener portrayed the way number as the total of the quantity of bonds between all combinations of particles [10], and is defined as pursues:

$$
W(G)=\frac{1}{2} \sum_{(u, v)} d(u, v)
$$

where $(u, v)$ is any discretionary match of vertices in $G$ and $d(u, v)$ is $u-v$ the length of most limited way among $u$ and $v$. The Eccentric-connectivity index $\xi(G)$ which was proposed by Sharma, Goswami and Madan characterized as [11]:

$$
\xi(G)=\sum_{u \in V(G)} d_{u} \epsilon_{u}
$$

Alaeiyan, Mojarad and Asadpour define the Eccentric connectivity polynomial of a graph $G$ as [12]:

$$
\operatorname{ECP}(G, x)=\sum_{u \in V(G)} d_{u} x^{\epsilon_{u}},
$$

where value of $x$ is greater than 1 . The relationship between eccentric connectivity polynomial and eccentric-connectivity index is given by

$$
\operatorname{ECP}(G, x)=\xi(G, 1),
$$

where $\xi(G, 1)$ is the principal subsidiary of $\operatorname{ECP}(G, x)$ [13]. Another essential Eccentricity based topological record is Connective Eccentric index $C^{\tilde{\xi}}(G)$ which was proposed by Gupta et al. [14]. The Connective Eccentric index is characterized as:

$$
C^{\xi}=\sum_{u \in V(G)} \frac{d_{u}}{\epsilon_{u}}
$$

For more information and properties of Eccentricity based topological index, see [15-22].

\section{Methods}

To compute our results, we utilized an approach for combinatorial enrolling, a vertex portion strategy, an edge allocate, outline hypothetical instruments, consistent structures, a degree-counting 
procedure, and a degrees of neighbors framework. Additionally, we used Matlab for intelligent estimations and attestations. We also utilized Maple for plotting numerical results.

\section{Eccentric Index of Crystal Structure of Cubic Carbon}

The structure of precious stone cubic carbon (or gem structure of cubic carbon ) is comprised of $3 \mathrm{D}$ squares and was talked about in [23]. The atomic chart of precious stone cubic carbon $C C C(n)$ for the first level is portrayed in Figure 1. For second level, new 3D shapes are joined at each end vertex of degree 3 of first level. The second level of $\operatorname{CCC}(n)$ is portrayed in Figure 2. Additionally, this method is rehashed to get the following level et cetera. The cardinality of vertices and edges in $\operatorname{CCC}(n)$ are given beneath separately.

$$
\begin{aligned}
& |V(\operatorname{CCC}(n))|=2\left\{24 \sum_{r=3}^{n}\left(2^{3}-1\right)^{r-3}+31\left(2^{3}-1\right)^{n-2}+2 \sum_{r=0}^{n-2}\left(2^{3}-1\right)^{r}+3\right\} . \\
& |E(\operatorname{CCC}(n))|=4\left\{24 \sum_{r=3}^{n}\left(2^{3}-1\right)^{r-3}+24\left(2^{3}-1\right)^{n-2}+2 \sum_{r=0}^{n-2}\left(2^{3}-1\right)^{r}+3\right\} .
\end{aligned}
$$

Let $Q_{k}$ be the set of all cubic free vertices in $\operatorname{CCC}(k)$ at the $k$-th level with $k \geq 2$, where we say that a vertex is cubic free if its degree is 3 at the $k$-th level. Let $v \in Q_{k}$, be an arbitrary element, in $(k+1)$-th level, we partition the new coming vertices in four sets. The number of new vertices at every $(k+1)$-th level is $448\left(2^{3}-1\right)^{k-3}$. The partition of these vertices is given as follows:

$S_{1}^{k}$ is the set of vertices at distance 1 from any $v \in Q_{k}$ with number of such vertices is $56\left(2^{3}-1\right)^{k-3}$, $S_{2}^{k}$ is the set of vertices at distance 2 from $v$ with cardinality $\left|S_{2}^{k}\right|=168\left(2^{3}-1\right)^{k-3}, S_{3}^{k}$ is the set of vertices at distance 3 from $v$ with cardinality $\left|S_{3}^{k}\right|=168\left(2^{3}-1\right)^{k-3}$ and $S_{4}^{k}$ is the set of vertices at distance 4 from $v$ with cardinality $\left|S_{4}^{k}\right|=56\left(2^{3}-1\right)^{k-3}$. In addition, we denote another set as $S^{k}$, which is the set of vertices of $c c c(1)$ that is the initial vertices in each $k$-th level, so cardinality $\left|S^{k}\right|=8$. This partition is also described in Table 1.

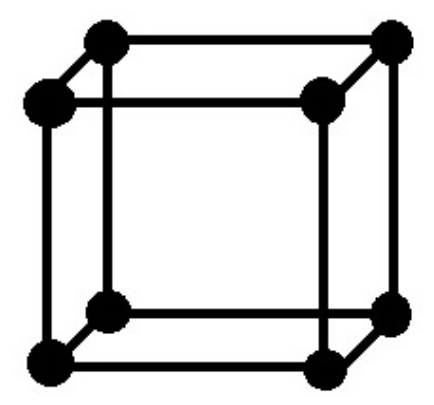

Figure 1. Crystal Structure Cubic Carbon CCC(1).

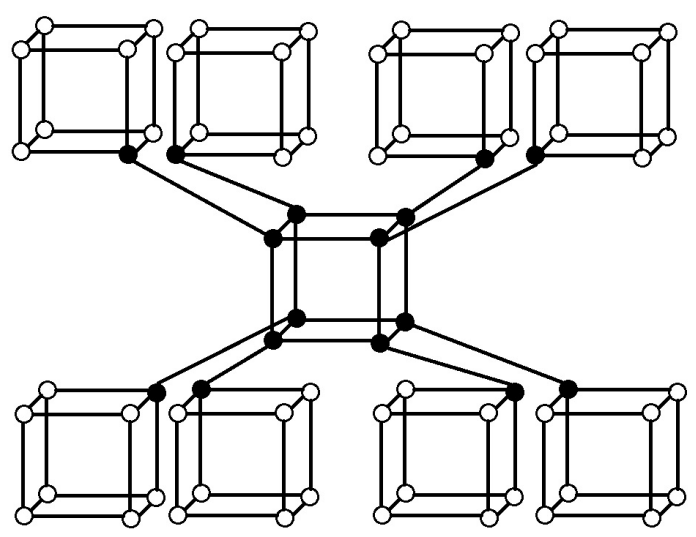

Figure 2. Crystal Structure Cubic Carbon CCC(2). 
Table 1. Vertex partition of new coming vertices at $(k+1)$-th level.

\begin{tabular}{ccc}
\hline Set & Number of Vertices & Distance from Arbitrary $v \in Q_{\boldsymbol{k}}$ \\
\hline$S_{1}$ & $56\left(2^{3}-1\right)^{k-3}$ & 1 \\
$S_{2}$ & $168\left(2^{3}-1\right)^{k-3}$ & 3 \\
$S_{3}$ & $168\left(2^{3}-1\right)^{k-3}$ & 3 \\
$S_{4}$ & $56\left(2^{3}-1\right)^{k-3}$ & 1 \\
\hline
\end{tabular}

In Table 2 we give the partition of all the vertices of $\operatorname{CCC}(n)$ based on degree, eccentricity and the membership to the sets $S_{1}, S_{2}, S_{3}$ and $S_{4}$.

Table 2. Vertex partition of $C C C(n)$ based on degree and eccentricity of each vertex.

\begin{tabular}{ccccc}
\hline Representative & Degree & Eccentricity & Frequency & Range \\
\hline$v \in S^{k}$ & 4 & $3+4(n-1)$ & 8 & $2 \leq k \leq n$ \\
$v \in S_{1}^{k}$ & 4 & $4(n+k-2)$ & $56\left(2^{3}-1\right)^{k-3}$ & $2 \leq k \leq n$ \\
$v \in S_{2}^{k}$ & 4 & $4(n+k)-7$ & $168\left(2^{3}-1\right)^{k-3}$ & $2 \leq k \leq n-1$ \\
$v \in S_{2}^{k}$ & 3 & $8 n-7$ & $168\left(2^{3}-1\right)^{k-3}$ & $k=n$ \\
$v \in S_{3}^{k}$ & 4 & $4(n+k)-6$ & $168\left(2^{3}-1\right)^{k-3}$ & $2 \leq k \leq n-1$ \\
$v \in S_{3}^{k}$ & 3 & $8 n-6$ & $168\left(2^{3}-1\right)^{k-3}$ & $k=n$ \\
$v \in S_{4}^{k}$ & 4 & $4(n+k)-5$ & $56\left(2^{3}-1\right)^{k-3}$ & $2 \leq k \leq n-1$ \\
$v \in S_{4}^{k}$ & 3 & $8 n-5$ & $56\left(2^{3}-1\right)^{k-3}$ & $k=n$ \\
\hline
\end{tabular}

In the coming Theorems, we have computed Eccentric-connectivity index $\xi(G)$, Eccentric connectivity polynomial $\operatorname{ECP}(G, x)$ and Connective Eccentric index $C^{\tau}(G)$ for crystal structure of cubic carbonCCC $(n)$.

Theorem 1. Consider the graph $G \cong C C C(n)$ with $n \geq 2$, then its Eccentric-connectivity index is equal to

$$
\xi(G)=\frac{\left(2^{6} \times 3 \times 7 \times 13 n-2^{5} \times 503\right) 7^{n}}{3^{2} \times 7^{2}}-\frac{2^{7}}{3} n+\frac{2^{5} \times 29}{3^{2}} .
$$

Proof. Let $G \cong C C C(n)$ be the crystal structure of cubic carbon. The vertex partition of $C C C(n)$ based on degrees of vertices and eccentricities with their frequencies is given in Table 2. Then by using Table 2 and Equation (1), the Eccentric-connectivity index can be calculated as follows:

$$
\begin{aligned}
\xi(G) & =\sum_{u \in V(G)} d_{u} \epsilon_{u} \\
\xi(G) & =8(4(3+4(n-1)))+4 \times \sum_{k=2}^{n} 56\left(2^{3}-1\right)^{k-3} 4(n+k-2) \\
& +4 \times \sum_{k=2}^{n-1} 168\left(2^{3}-1\right)^{k-3}(4(n+k)-7)+3 \times 168\left(2^{3}-1\right)^{n-3}(8 n-7) \\
& +4 \times \sum_{k=2}^{n-1} 168\left(2^{3}-1\right)^{k-3}(4(n+k)-6)+3 \times 168\left(2^{3}-1\right)^{n-3}(8 n-6) \\
& +4 \times \sum_{k=2}^{n-1} 56\left(2^{3}-1\right)^{k-3}(4(n+k)-5)+3 \times 56\left(2^{3}-1\right)^{n-3}(8 n-5)
\end{aligned}
$$

After, some easy computations, we get:

$$
\xi(G)=\frac{\left(2^{6} \times 3 \times 7 \times 13 n-2^{5} \times 503\right) 7^{n}}{3^{2} \times 7^{2}}-\frac{2^{7}}{3} n+\frac{2^{5} \times 29}{3^{2}} .
$$


The 3D-graphical representation of the Eccentric-connectivity index is depicted in Figure 3.

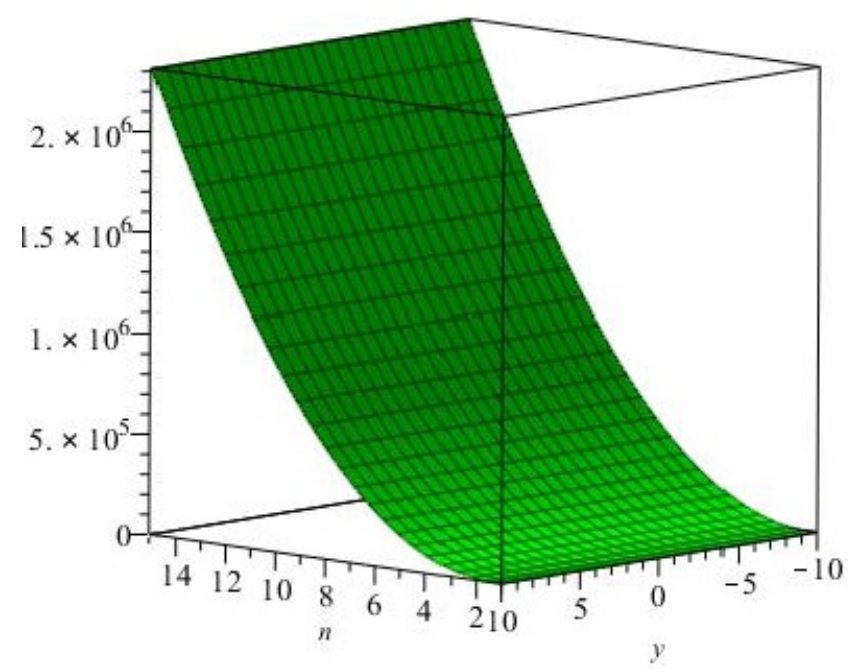

Figure 3. The graphical representation of the Eccentric-connectivity index.

Theorem 2. Consider the graph $G \cong \operatorname{CCC}(n)$ with $n \geq 2$, then its Eccentric connectivity polynomial is equal to

$$
\begin{aligned}
E C P(G, x) & =32 x^{(4 n-1)}+168(7)^{n-3} x^{8 n-5}+504(7)^{n-3} x^{8 n-6}+504(7)^{n-3} x^{8 n-7} \\
& +224 \times \sum_{k=2}^{n}(7)^{k-3} x^{4(n+k-2)}+672 \times \sum_{k=2}^{n-1}(7)^{k-3} x^{4(n+k)-7} \\
& +672 \times \sum_{k=2}^{n-1}(7)^{k-3} x^{4(n+k)-6}+224 \times \sum_{k=2}^{n-1}(7)^{k-3} x^{4(n+k)-5} .
\end{aligned}
$$

Proof. Let $G \cong C C C(n)$ be the crystal structure of cubic carbon. Then by using Table 2 and Equation (2) the Eccentric connectivity polynomial is given by:

$$
\begin{aligned}
\operatorname{ECP}(G, x) & =\sum_{u \in V(G)} d_{u} x^{\epsilon_{u}} \\
\operatorname{ECP}(G, x) & =8 \times 4 x^{3+4(n-1)}+4 \times \sum_{k=2}^{n} 56\left(2^{3}-1\right)^{k-3} x^{4(n+k-2)} \\
& +4 \times \sum_{k=2}^{n-1} 168\left(2^{3}-1\right)^{k-3} x^{4(n+k)-7}+3 \times 168\left(2^{3}-1\right)^{n-3} x^{8 n-7} \\
& +4 \times \sum_{k=2}^{n-1} 168\left(2^{3}-1\right)^{k-3} x^{4(n+k)-6}+3 \times 168\left(2^{3}-1\right)^{n-3} x^{8 n-6} \\
& +4 \times \sum_{k=2}^{n-1} 56\left(2^{3}-1\right)^{k-3} x^{4(n+k)-5}+3 \times 56\left(2^{3}-1\right)^{n-3} x^{8 n-5} .
\end{aligned}
$$

After an easy calculation, we get:

$$
\begin{aligned}
E C P(G, x) & =32 x^{(4 n-1)}+168(7)^{n-3} x^{8 n-5}+504(7)^{n-3} x^{8 n-6}+504(7)^{n-3} x^{8 n-7} \\
& +224 \times \sum_{k=2}^{n}(7)^{k-3} x^{4(n+k-2)}+672 \times \sum_{k=2}^{n-1}(7)^{k-3} x^{4(n+k)-7} \\
& +672 \times \sum_{k=2}^{n-1}(7)^{k-3} x^{4(n+k)-6}+224 \times \sum_{k=2}^{n-1}(7)^{k-3} x^{4(n+k)-5} .
\end{aligned}
$$


The 3D-graphical representation of the Eccentric connectivity polynomial is depicted in Figure 4.

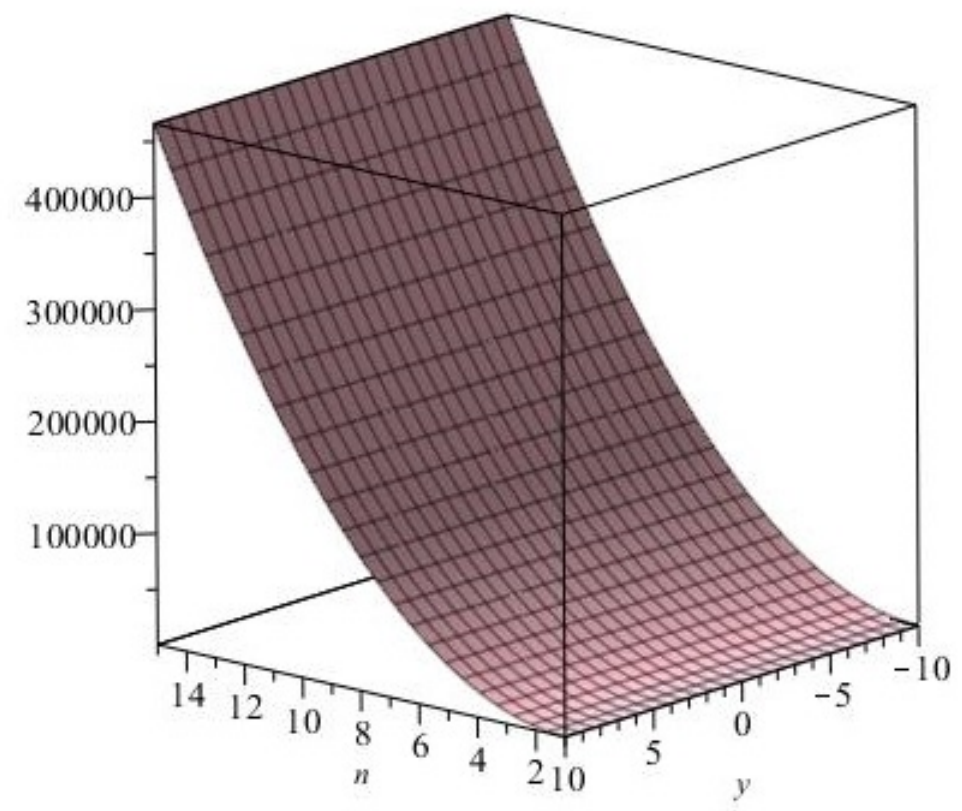

Figure 4. The graphical representation of the Eccentric connectivity polynomial.

Theorem 3. Consider the graph $G \cong C C C(n)$ with $n \geq 2$, then its Connective Eccentric index is equal to

$$
\begin{aligned}
C^{\xi}(G) & =\frac{32}{(4 n-1)}+\frac{727^{n}}{(392 n-343)}+\frac{367^{n}}{(196 n-147)}+\frac{247^{n}}{(392 n-245)} \\
& +224 \times \sum_{k=2}^{n} \frac{(7)^{k-3}}{4(n+k-2)}+672 \times \sum_{k=2}^{n-1} \frac{(7)^{k-3}}{4(n+k)-7}+672 \times \sum_{k=2}^{n-1} \frac{(7)^{k-3}}{(4(n+k)-6)} \\
& +224 \times \sum_{k=2}^{n-1} \frac{(7)^{k-3}}{4(n+k)-5} .
\end{aligned}
$$

Proof. Let $G \cong C C C(n)$ be the crystal structure of cubic carbon. Then by using Table 2 and Equation (4) the Connective Eccentric index is calculated as:

$$
\begin{aligned}
C^{\xi}(G) & =\sum_{u \in V(G)} \frac{d_{u}}{\epsilon_{u}} \\
C^{\xi}(G) & =\frac{32}{3+4(n-1)}+4 \times \sum_{k=2}^{n} \frac{56\left(2^{3}-1\right)^{k-3}}{4(n+k-2)}+4 \times \sum_{k=2}^{n-1} \frac{168\left(2^{3}-1\right)^{k-3}}{4(n+k)-7} \\
& +168\left(2^{3}-1\right)^{n-3} \times \frac{3}{8 n-7}+4 \times \sum_{k=2}^{n-1} \frac{168\left(2^{3}-1\right)^{k-3}}{(4(n+k)-6)}+168\left(2^{3}-1\right)^{n-3} \times \frac{3}{8 n-6} \\
& +4 \times \sum_{k=2}^{n-1} \frac{56\left(2^{3}-1\right)^{k-3}}{4(n+k)-5}+56\left(2^{3}-1\right)^{n-3} \times \frac{3}{8 n-5}
\end{aligned}
$$


After an easy calculation, we get:

$$
\begin{aligned}
C^{\xi}(G) & =\frac{32}{(4 n-1)}+\frac{727^{n}}{(392 n-343)}+\frac{367^{n}}{(196 n-147)}+\frac{247^{n}}{(392 n-245)} \\
& +224 \times \sum_{k=2}^{n} \frac{(7)^{k-3}}{4(n+k-2)}+672 \times \sum_{k=2}^{n-1} \frac{(7)^{k-3}}{4(n+k)-7}+672 \times \sum_{k=2}^{n-1} \frac{(7)^{k-3}}{(4(n+k)-6)} \\
& +224 \times \sum_{k=2}^{n-1} \frac{(7)^{k-3}}{4(n+k)-5} .
\end{aligned}
$$

The 3D-graphical representation of the Connective Eccentric index is depicted in Figure 5.

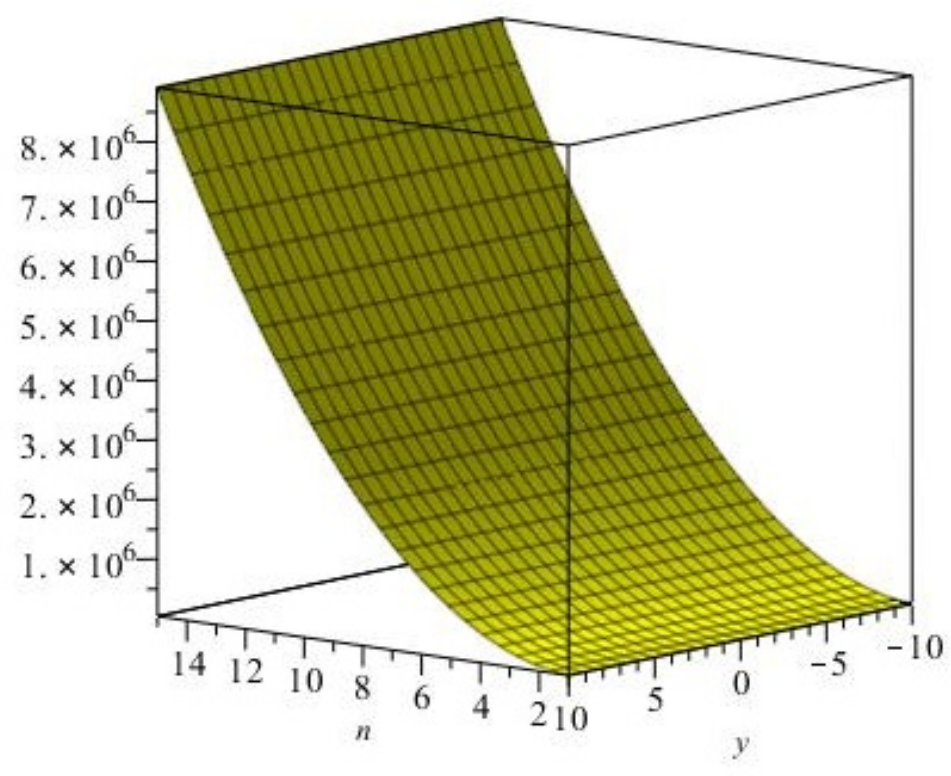

Figure 5. The graphical representation of the Connective Eccentric index.

\section{Numerical and Graphical Comparison of Computed Indices}

In this section we shall compare Eccentric-connectivity index, Eccentric connectivity polynomial and Connective Eccentric index of $\operatorname{CCC}(n)$, both numerically and graphically. The Table 3 shows a numerical comparison of the described indices and the polynomial. The graphical representation of the numerical comparison is depicted in Figure 6.

Table 3. Comparison of the $\xi(G), C^{\xi}(G)$ and $\operatorname{ECP}(G, x)$ of $G \cong C C C(n)$.

\begin{tabular}{llll}
\hline$n$ & $\xi(G)$ & $C^{\xi}(G)$ & $\begin{array}{l}E C P(G, x) \\
\text { Approx. }\end{array}$ \\
\hline 2 & 5457.60 & 9352 & $32,038.4$ \\
3 & $533,337.60$ & 538,880 & $3,224,281.6$ \\
4 & $4,183,459.20$ & $31,716,640$ & $242,651,430.4$ \\
5 & $30,899,311.808$ & $2,065,600,096$ & $1,612,928,748.80$ \\
6 & $22,891,814.30464$ & $62,224,801,952$ & $100,242,075,452.16$ \\
7 & $8,645,581,788.7936$ & $7,559,088,080,480$ & $5,974,731,403,050.24$ \\
8 & $541,359,817,758.7904$ & $225,395,825,441,440$ & $346,142,855,211,733.76$ \\
9 & $34,985,496,157,764.4224$ & $7,144,399,013,120,096$ & $196,480,917,540,735.5008$ \\
10 & $239,376,206,786,041.20768$ & $401,075,576,608,315,552$ & $10,983,346,993,039,897.4848$ \\
\hline
\end{tabular}


The next graph shows the graphical comparison of Eccentric-connectivity index, Eccentric connectivity polynomial and Connective Eccentric index of $\operatorname{CCC}(n)$.

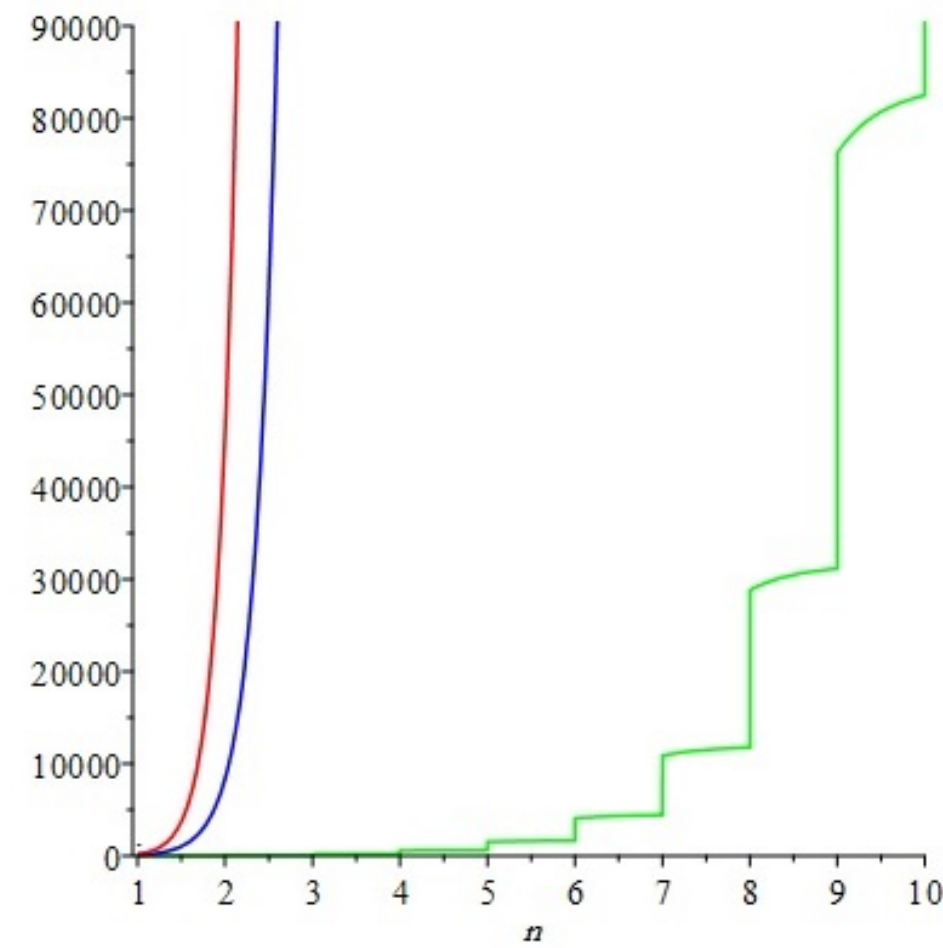

Figure 6. Graphical Comparison of indices of $\operatorname{CCC}(1) ; \xi(G)$ is red, $C^{\tau}(G)$ is blue, and $\operatorname{ECP}(G, x)$ is green.

\section{Conclusions}

In this article, we have discussed the Eccentric Connectivity index, Eccentric connectivity polynomial and Connective Eccentric index. We consider the molecular graph of crystal structure cubic carbon $C C C(n)$ and we have computed Connective Eccentric index, Eccentric connectivity polynomial and Connective Eccentric index of crystal structure of cubic carbon $C C C(n)$ chemical graphs for $n$-levels.

Author Contributions: H.Y. contribute for supervision, project administration, funding and analyzed the data curation. M.K.S. and M.A. contribute for designing the experiments, validation, conceptualization, formal analysing experiments, resources, software and some computations. M.N. contribute for Investigation, methodology and wrote the initial draft of the paper which were investigated and approved by M.K.S. and wrote the final draft. All authors read and approved the final version of the paper.

Funding: This work was supported by the Soft Scientific Research of Sichuan Province under grant 2018ZR0265, Sichuan Military and Civilian Integration Strategy Research Center under grant JMRH-1818, and Sichuan Provincial Department of Education (Key Project) under grant 18ZA0118.

Acknowledgments: The authors are grateful to the anonymous referee for their valuable comments and suggestions that improved this paper.

Conflicts of Interest: The authors declare no conflict of interest.

\section{References}

1. Balaban, A.T.; Quintas, L.V. The smallest graphs, trees, and 4-trees with degenerate topological index. J. Math. Chem. 1983, 14, 213-233.

2. Graovac, A.; Ghorbani, M.; Hosseinzadeh, M.A. Computing fifth geometric-arithmetic index for nanostar dendrimers. J. Math. Nanosci. 2011, 1,33-42.

3. Gutman, I.; Polansky, O.E. Mathematical Concepts in Organic Chemistry; Springer: New York, NY, USA, 1986. 
4. Randić, M. On characterization of molecular branching. J. Am. Chem. Soc. 1975, 97, 6609-6615. [CrossRef]

5. Gutman, I.; Trinajstć, N. Graph theory and molecular orbitals., Total $\pi$-electron energy of alternant hydrocarbons. Chem. Phys. Lett. 1972, 17, 535-538. [CrossRef]

6. Bonchev, D. Handbook of Graph Theory, Chemical Graph Theory; Virginia Commonwealth University: Richmond, VA, USA, 2013; Section 13.

7. Asadpour, J.; Safikhani, L. Study of $\mathrm{CNC}_{7}[n]$ Carbon Nanocone by M-Eccentric Connectivity Polynomial. Aust. J. Basic Appl. Sci. 2013, 7, 883.

8. De, N.; Nayeem, S.M.A.; Pal, A. Total eccentricity index of the generalized index and polynomial of thorn graph. Appl. Math. 2012, 3, 931-934. [CrossRef]

9. Huo, Y.; Lio, J.B.; Baig, A.Q.; Sajjad, W.; Farahani, M.H. Connective Eccentric Index of $N A_{m}^{n}$ Nanotube. J. Comput. Theor. Nanosci. 2017, 14, 1832-1836. [CrossRef]

10. Wiener, H. Structural determination of paraffin boiling points. J. Am. Chem. Soc. 1947, 69, 17-20. [CrossRef] [PubMed]

11. Sharma, V.; Goswami, R.; Madan, A.K. Eccentric connectivity index: A novel highly discriminating topological descriptor for structure-property and structure-activity studies. J. Chem. Inf. Comput. Sci. 1997, 37, $273-282$. [CrossRef]

12. Alaeiyan, M.; Mojarad, R.; Asadpour, J. A new method for computing eccentric connectivity polynomial of an infinite family of linear polycene parallelogram benzenod. Optoelectron. Adv. Mater.-Rapid Commun. 2011, $5,761-770$.

13. Bindusree, A.R.; Lokesha, V.; Ranjini, P.S. Eccentric connectivity index and polynomial of some graphs. Br. J. Math. Comput. Sci. 2015, 6, 457-467. [CrossRef]

14. Gupta, S.; Singh, M.; Madan, A.K. Connective eccentricity index: a novel topological descriptor for predicting biological activity. J. Mol. Graph Model 2000, 18, 18-25. [CrossRef]

15. Gupta, S.; Singh, M.; Madan, A.K. Application of Graph Theory: Relationship of Eccentric Connectivity Index and Wiener's Index with Anti-inflammatory Activity. J. Math. Anal. Appl. 2002, 266, 259-268. [CrossRef]

16. Zhang, X.; Siddiqui, M.K.; Naeem, M.; Baig, A.Q. Computing Eccentricity Based Topological Indices of Octagonal Grid. Mathematics 2018, 6, 153. [CrossRef]

17. Siddiqui, M.K.; Naeem, M.; Rahman, N.A.; Imran, M. Computing topological indicesof certain networks. J. Optoelectron. Adv. Mater. 2016, 18, 884-892.

18. Siddiqui, M.K.; Imran, M.; Ahmad, A. On Zagreb indices, Zagreb polynomials of some nanostar dendrimers. Appl. Math. Comput. 2016, 280, 132-139. [CrossRef]

19. Naeem, M.; Siddiqui, M.K.; Guirao, J.L.G.; Gao, W. New and Modified Eccentric Indices of Octagonal Grid. Appl. Math. Nonlinear Sci. 2018, 3, 209-228. [CrossRef]

20. Imran, M.; Siddiqui, M.K.; Abunamous, A.A.E.; Adi, D.; Rafique, S.H.; Baig, A.Q. Eccentricity Based Topological Indices of an Oxide Network. Mathematics 2018, 6, 126. [CrossRef]

21. Ramane, H.S.; Jummannaver, R.B. Note on forgotten topological index of chemical structure in drugs. Appl. Math. Nonlinear Sci. 2016, 1, 369-374. [CrossRef]

22. Gao, W.; Imran, M.; Siddiqui, M.K.; Naeeme, M.; Jamil, F. Molecular Description of Copper (I) Oxide and Copper (II) Oxide. Quimica Nova 2018, 41, 874-879. [CrossRef]

23. Baig, A.Q.; Imran, M.; Khalid, W.; Naeem, M. Molecular description of carbon graphite and crystal cubic carbon structures. Can. J. Chem. 2017, 95, 674-686. [CrossRef]

(c) 2018 by the authors. Licensee MDPI, Basel, Switzerland. This article is an open access article distributed under the terms and conditions of the Creative Commons Attribution (CC BY) license (http:/ / creativecommons.org/licenses/by/4.0/). 